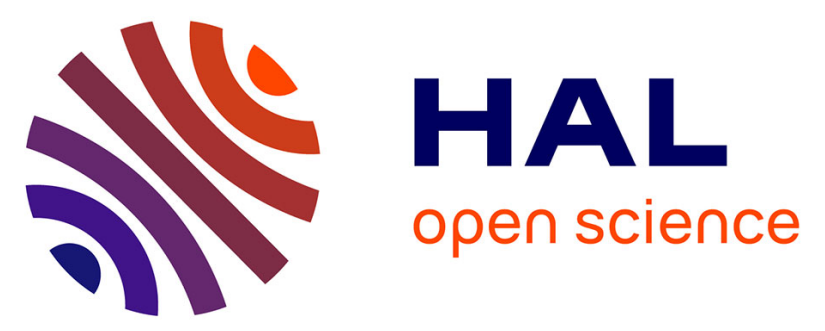

\title{
Single-Mode Fiber Coupling with Adaptive Optics for Free-Space Optical Communication under Strong Scintillation
}

C B Lim, Aurélie Montmerle-Bonnefois, Cyril Petit, Jean-Francois Sauvage, Serge Meimon, Philippe Perrault, Francis Mendez, Bruno Fleury, Joseph Montri, Jean-Marc Conan, et al.

\section{To cite this version:}

C B Lim, Aurélie Montmerle-Bonnefois, Cyril Petit, Jean-Francois Sauvage, Serge Meimon, et al.. Single-Mode Fiber Coupling with Adaptive Optics for Free-Space Optical Communication under Strong Scintillation. 2019 IEEE International Conference on Space Optical Systems and Applications (ICSOS), Oct 2019, Portland, United States. 10.1109/ICSOS45490.2019.8978978 . hal-02506513

\section{HAL Id: hal-02506513 https://hal.science/hal-02506513}

Submitted on 30 Nov 2020

HAL is a multi-disciplinary open access archive for the deposit and dissemination of scientific research documents, whether they are published or not. The documents may come from teaching and research institutions in France or abroad, or from public or private research centers.
L'archive ouverte pluridisciplinaire HAL, est destinée au dépôt et à la diffusion de documents scientifiques de niveau recherche, publiés ou non, émanant des établissements d'enseignement et de recherche français ou étrangers, des laboratoires publics ou privés. 


\section{Single-mode fiber coupling with adaptive optics for free-space optical communication under strong scintillation}

Caroline B. Lim, Aurélie Montmerle-Bonnefois, Cyril Petit, Jean-François Sauvage, Serge Meimon, Philippe Perrault, Francis Mendez, Bruno Fleury, Joseph Montri, Jean-Marc Conan, Vincent Michau and Nicolas Védrenne

ONERA, DOTA

Paris Saclay University

92322 Châtillon, France

caroline.lim@,onera.fr

\begin{abstract}
The need for space-ground data transmission systems with high data throughput has encouraged the development of new satellite-to-ground laser links, using adaptive optics to compensate for the deleterious effects of atmospheric turbulence. On the ground segment, the use of single-mode fiber components, and thus the coupling of the propagating signal into a single-mode fiber, is strongly favored. However, the single-mode fiber coupling efficiency and the resulting data rate are strongly hampered by the turbulenceinduced phase distortions and amplitude fluctuations (called "scintillation"). The effects of scintillation in the pupil are the strongest at low elevations of Low Earth Orbit satellites-toground links and are not compensated by traditional adaptive optics, which correct only the phase of the distorted signal. In this paper, we assess the effects of AO correction on the statistical properties of single-mode fiber coupled flux based on experimental data obtained in the presence of medium and strong scintillation. We show preliminary results of coupled flux, as well as their temporal variations and probability distribution, for different adaptive optics correction levels.
\end{abstract}

Keywords-adaptive optics, free-space optical communication, single-mode fiber coupling

\section{INTRODUCTION}

Next generation satellite-to-ground laser communication systems are identified as a promising alternative to radiofrequency links to match the future need for space-ground data transmission with very high data rates. Indeed, shifting the communication channels to the optical frequency domain offer several advantages: potentially much higher data rates, a regulation free spectrum, the availability of mature optical fibered-components for the ground segment (detectors, multiplexers, amplifiers), and an enhanced privacy of the transmission channel provided by the intrinsic directivity of the laser beam. However, the atmospheric turbulence severely degrades the spatial coherence of the propagating signal, which results in signal phase distortions and amplitude fluctuations in the pupil (called scintillation). Considering a satellite-to-ground downlink, this results in a collapse of the single-mode fiber (SMF) coupling of the free-space optical signal at the ground receiver end, which is disastrous for the link budget.

The compensation of the turbulence-induced phase distortions is possible using adaptive optics (AO) ahead of the SMF coupling. Originally developed for astronomy, this technique has recently raised an increasing interest for its potential application in free space optical communications.
Intensive research is especially being carried out in the framework of Low Earth Orbit (LEO) satellite-to-ground optical links. Indeed, as compared to astronomy observation or telecommunication links with geostationary (GEO) satellites cases, the LEO-ground scenarios involve atmospheric turbulence conditions specifically demanding regarding the $\mathrm{AO}$ robustness to strong scintillation and high temporal dynamics. Several in-lab demonstrations were realized in the last 10 years [1]-[3] and paved the way towards an on-sky experimental optical link. In 2015, NASA reported the first ground-space AO-assisted SMF injection of an optical downlink with the International Space Station using the Optical Payload for Lasercomm Science [4], and a French-Japanese collaboration reported the first microsatellite-to-ground optical downlink with AO correction using the SOTA terminal onboard SOCRATES micro-satellite [5].

The FEEDELIO experiment [6], [7] originally aims at demonstrating the feasibility and quantifying the limits of pre-compensation of a ground-to-GEO satellite feeder link using AO in representative experimental conditions. A bidirectional link is established on a $13 \mathrm{~km}$ slant path $(\sim 5$ degrees) between Mount Teide (altitude $3500 \mathrm{~m}$ ) and the ESA Optical Ground Station (OGS, altitude $2400 \mathrm{~m}$ ), in Canary Island, Tenerife. On Mount Teide, a module emulating a satellite receives and detects the precompensated uplink signal. On the ground, the OGS hosts our telescope (diameter $35 \mathrm{~cm}$ ) coupled to our AO-bench, which is equipped with a SMF injection module of the downlink signal. This provides the AO-corrected downlink data at the heart of this study. These data are somewhat relevant regarding a low elevation LEO-to-ground link in terms of scintillation conditions.

In this short paper, we assess the effect of AO correction on the statistical properties of SMF coupling performed in a satellite-to-ground scenario in the presence of scintillation. We compare the results obtained for two sets of data acquired under medium and strong scintillation conditions, but similar Fried and wind speed parameters.

In a first part, we describe the downlink part of the FEEDELIO experimental setup, including the AO and SMF injection modules. In a second part, we estimate the atmospheric turbulence conditions main parameters based on the wavefront sensing experimental data. In a third part, we show preliminary results of AO correction performance and resulting downlink SMF coupling statistics. 


\section{EXPERIMENTAL SETUP}

Fig. 1 (up) illustrates the FEEDELIO downlink line of sight, where the satellite module on Mount Teide points towards the telescope and transmits a downlink $1.55 \mu \mathrm{m}$ laser signal to the AO-bench. The downlink signal is transmitted by the telescope and enters the AO bench presented in Fig. 1 (down) by the upper left side of the image. The common optical path is represented in yellow. The signal optical path then reflects on the surface of the DM, which corrects the wavefront, and is then used both to assess the turbulenceinduced phase distortion (green path) via measurement by a Shack-Hartmann wavefront sensor (WFS) and to perform the SMF coupling (red path). The SMF module is optically aligned with respect to the optical axis before the data acquisition using an internal laser source. A focal plane camera (FPC) is used to visualize the point spread function (PSF) (blue path). A real-time calculator (RTC) computes the correction phase to be applied by the DM based on the WFS measurements with respect to a reference wavefront. More details on the design of the set-up can be found in [6].
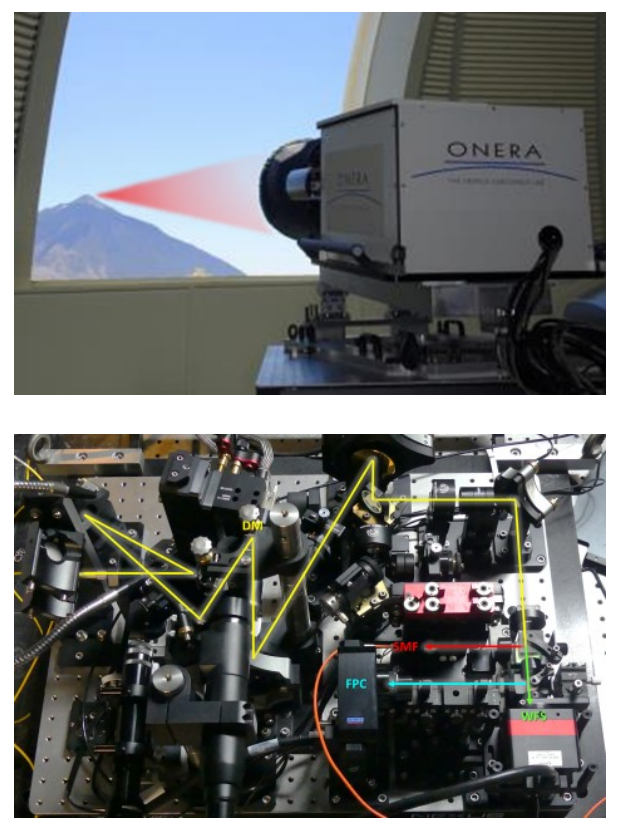

Fig. 1. Up: The FEEDELIO experiment between Mount Teide and the OGS. The AO-bench is located inside the ONERA box. Down: The AObench, including DM and WFS and equipped with a downlink SMF injection module.

In terms of experimental parameters, the telescope has a pupil of diameter $35 \mathrm{~cm}$, with a central occultation of $23 \%$. The Shack-Hartmann WFS is composed of an $8 \times 8$ microlenses matrix coupled to a RAPTOR CMOS camera in their focal plane. The DM is composed of 97 actuators (Alpao 11x11 Cartesian grid). The AO control loop running at a sampling frequency of $1.5 \mathrm{kHz}$ with a loop delay of 2.3 frames is closed with an integrated controller gain of 0.5 . The SMF coupled flux is measured by an InGaAs PIN monodetector recording $10 \mathrm{~s}$ series sampled at $20 \mathrm{kHz}$. The FPC registers $64 \times 64$ pixels images stacks of total duration pose $1 \mathrm{~s}$ sampled at $1 \mathrm{kHz}$. They enable to visualize the PSF, providing a real-time AO-performance indicator.
Each set of data includes temporal series of WFS intensities and slopes, SMF coupled flux and FPC images, all acquired for three AO configurations: open loop (no correction), tiptilt only correction (also referred to as 1 radial order correction), full AO correction.

To evaluate the SMF coupling efficiency, the SMF coupled flux is normalized by the flux integrated on the FPC images recorded simultaneously. This normalization neutralizes the pupil-averaged intensity fluctuation, but the effect of the scintillation induced inhomogeneous irradiance inside the pupil remains.

In the following, we focus on two sets of data acquired on April 14 ${ }^{\text {th }} 2019$ at 09:28 pm (data 1) and 10:55 pm (data 2), with strong and medium scintillation conditions respectively.

\section{ESTIMATION OF THE TURBULENCE CONDITIONS}

In this part, we detail the method used to assess the turbulence conditions relative to the data sets 1 and 2 , and show the resulting parameters estimations. We choose to describe the turbulence using the three main parameters: Fried parameter $r_{0}$ at $1.55 \mu \mathrm{m}$ on the line of sight, wind speed parameter $S_{\text {wind }}$, and log-amplitude fluctuation per sub-aperture $\sigma_{\chi}^{2}$ (also called "scintillation").

The Shack-Hartmann WFS performs a spatial sampling of the wavefront. The local slope of the turbulent wavefront sampled by each sub-aperture is estimated based on the position of the focal spot compared to that obtained for the reference wavefront. A phase reconstruction on the 54 first Zernike polynomials is computed from the pseudo openloop Shack-Hartmann WFS $\mathrm{x}$ and $\mathrm{y}$ slopes. The Fried parameter is estimated on the obtained Zernike coefficient variances. 
(a) Data 1

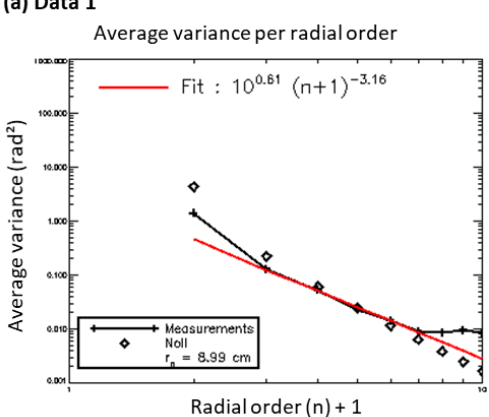

(b) Data 2

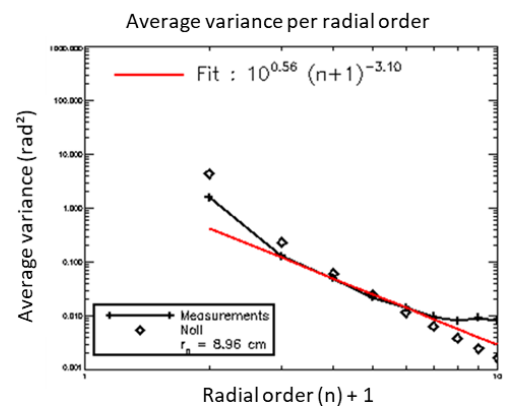

Fig. 2. Average residual phase variance per radial order for (a) data 1, (b) data 2, estimated from WFS slopes data. Variances estimated from our measurements are in black solid line with plus signs, variances estimated from Noll formalism are in diamonds, and experimental fits with a power law are in red solid line.

Fig. 2 (a) and (b) show the average variances estimated per radial order for data 1 and data 2 , respectively. Similar to [5], only the radial orders 3 to 6 are used for this estimation: the first two radial orders are sensitive to vibrations and outer scale (not estimated here), and radial orders higher than 6 are sensitive to aliasing errors. The estimated Fried parameter $r_{0}$ is the same for data 1 and data $2: r_{0}=9 \mathrm{~cm}$.

To estimate the wind speed, we exploit the temporal Power Spectral Density (PSD) of the $\mathrm{x}$ and $\mathrm{y}$ slopes measured by the WFS in each sub-aperture. The asymptotic fits of these PSD cross at the frequency defined by [8]:

$$
f=0.3 s_{\text {wind }} / d_{\text {sub }}
$$

where $s_{\text {wind }}$ is the averaged wind speed parameter and $d_{\text {sub }}$ is the sub-pupil diameter. This is illustrated in Fig. 3 (a) and (b) for data 1 and data 2, where the intersection frequencies are $\sim 20 \mathrm{~Hz}$ and $\sim 18 \mathrm{~Hz}$, and the estimated wind speed parameters are thus very close: $1.7 \mathrm{~m} / \mathrm{s}$ and $2.0 \mathrm{~m} / \mathrm{s}$, respectively.

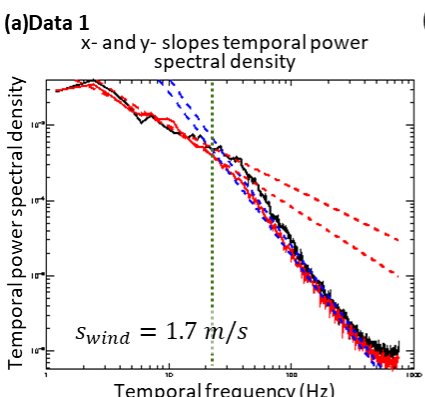

(b)Data 2

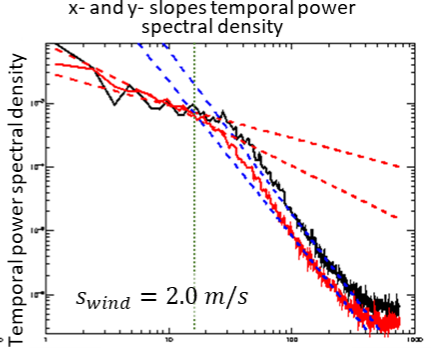

Temporal frequency $(\mathrm{Hz})$

Fig. 3. x- (black), y-slopes (red) temporal power spectral densities and respective asymptotic fits (dashed lines), for (a) data 1 and (b) data 2. The green dotted line shows the intersection frequency of the fits.
The log-amplitude variances averaged on the sub-apertures are estimated based on the WFS intensities recorded for data 1 and data 2 respectively: $\sigma_{\chi_{1}}^{2}=0.43$ and $\sigma_{\chi_{2}}^{2}=0.14$.

The log-amplitude variance for data 1 is 3 times larger than that for data 2. This confirms the stronger scintillation encountered for data 1 , which acquisition was indeed performed earlier in the evening.

These parameters were estimated for the entire sets of data acquired on-site. As a result, Fig. 4 illustrates the large diversity of the turbulence conditions encountered on April $14^{\text {th }} 2019$ between $9 \mathrm{pm}$ and $12 \mathrm{pm}$. The Fried parameters and log-amplitude variances are respectively $r_{0}=9.1 \pm 1.8 \mathrm{~cm}$ and $\sigma_{x}^{2}=0.33 \pm 0.15$
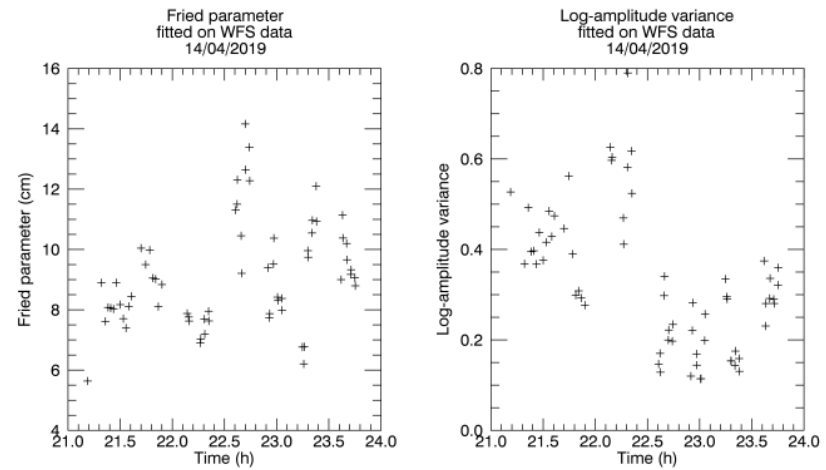

Fig. 4. Fried parameter and Log-Amplitude variance estimated from the WFS data.

Similarly, Fig. 5 shows the estimated speeds of wind encountered on April $14^{\text {th }} 2019$ between $9 \mathrm{pm}$ and $12 \mathrm{pm}: 2.32 \pm 0.98 \mathrm{~m} / \mathrm{s}$. TABLE 1 sums up the turbulence parameters.

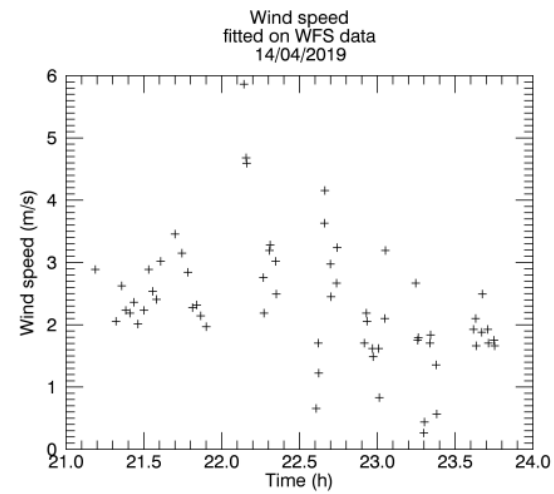

Fig. 5. Wind speed deduced from the WFS slopes data.

TABLE 1. TURBULENCE CONDITIONS: FRIED PARAMETER $r_{0}$, SPEED OF WIND $s_{\text {wind }}$, LOG-AMPLITUDE VARIANCE $\sigma_{\chi}^{2}$.

\begin{tabular}{|c|c|c|c|}
\hline & Data 1 & Data 2 & 9-12 pm \\
\hline $\mathbf{r}_{\mathbf{0}}(\mathbf{c m})$ & 9.0 & 9.0 & $9.1 \pm 1.8$ \\
\hline $\mathbf{s}_{\text {wind }}(\mathbf{m} / \mathbf{s})$ & 1.7 & 2.0 & $2.3 \pm 0.98$ \\
\hline $\boldsymbol{\sigma}_{\chi}^{2}$ & 0.43 & 0.14 & $0.33 \pm 0.15$ \\
\hline
\end{tabular}

IV. EXPERIMENTAL RESULTS OF SINGLE-MODE FIBER COUPLING 
In this part, we present the results of downlink single-mode fiber coupling for data 1 and data 2, jointly with an evaluation of the AO correction performance. Mean coupling efficiencies and temporal variations of the coupled flux are assessed.

\section{A. Performance of the AO correction}

The performance of the AO correction can be assessed by estimating the Strehl ratio on the PSF images acquired by the FPC.

Using an internal source at the telescope focal point, the optimized internal Strehl ratio was measured and reached the maximum value of $\mathrm{SR}_{0}=85 \%$. In practice, this ideal Strehl ratio is however degraded by AO-related error terms which must be accounted for. Based on the AO error budget presented in [9], the dominating error term is the fitting error $\sigma_{\text {fitting }}^{2}$, estimated by:

$$
\sigma_{\text {fitting }}^{2}=0.458\left(n_{\max }+1\right)^{-5 / 3}\left(D / r_{0}\right)^{5 / 3}
$$

where $n_{\max }=5$ is the highest corrected radial order.

Accounting for the log-amplitude variance estimated on the WFS data as described in [10], this leads to a maximum Strehl ratio estimation of:

$$
\mathrm{SR} \sim \mathrm{SR}_{0} \exp \left(-\sigma_{\text {fitting }}^{2}-\sigma_{\chi}^{2}\right)
$$

i.e. maximum Strehl ratio of:

$\mathrm{SR}_{1}=52.3 \%$ and $\mathrm{SR}_{2}=69.5 \%$ estimated for data 1 and 2 respectively. The Fried parameters being the same for both data sets, the fitting errors are the same too. As a result, the phase correction quality performed by the $\mathrm{AO}$ is expected to be similar, so that the difference between $\mathrm{SR}_{1}$ and $\mathrm{SR}_{2}$ should only lie in the log-amplitude variance terms. This is indeed numerically verified:

$$
\mathrm{SR}_{1}=\mathrm{SR}_{2} \exp \left(-\sigma_{\chi_{1}}^{2}+\sigma_{\chi_{2}}^{2}\right)
$$

Fig. 6 shows the PSF images for data 1 (pink) and data 2 (purple), acquired with an exposure time of $1 \mathrm{~s}$ (long with respect to the turbulence temporal dynamics) under different AO correction configurations. The corresponding Strehl ratio are indicated below each image. The open loop Strehl ratios are similar for both data sets, with a slightly lower one for the strong scintillation case. This is consistent with the turbulence conditions being the same, except for scintillation, for both data sets. Data 2 displays the highest Strehl ratio with full AO correction with a value of $40.5 \%$, which is still $42 \%$ lower than estimated by (3). Similarly, the full correction Strehl ratio for data 1 is $33.3 \%$, i.e. $36 \%$ lower than estimated by (3). On the one hand, the experimental Strehl ratio values verify the equation (4). This points out that the phase correction performed by our AO system is not affected by the considered intensity inhomogeneity in the pupil. The optical quality (Strehl ratio) obtained in full AO correction is still better in the case of weaker scintillation, and is thus degraded by the pupil intensity inhomogeneity. On the other hand, comparing these experimental values to our estimations using equation (3) reveals a mismatch that could be attributed to the apparent defocus on the PSF images. Indeed, the acquisitions were affected by a varying defocus aberration of peak-valley amplitude $1 \mathrm{rad}$. This raises the issue of the non-common path aberrations (NCPAs), which were not minimized due to the complexity of the overall experiment and were indeed significant during the data acquisitions. This could contribute heavily to the mismatch between the Strehl ratio measured and expected, and will be further assessed in future work.

The gain in Strehl ratio between two AO corrections is also detailed in the figure. The gain brought by the tip-tilt correction and by the full AO correction respectively are slightly higher for data 2 than for data 1 , but remain comparable.

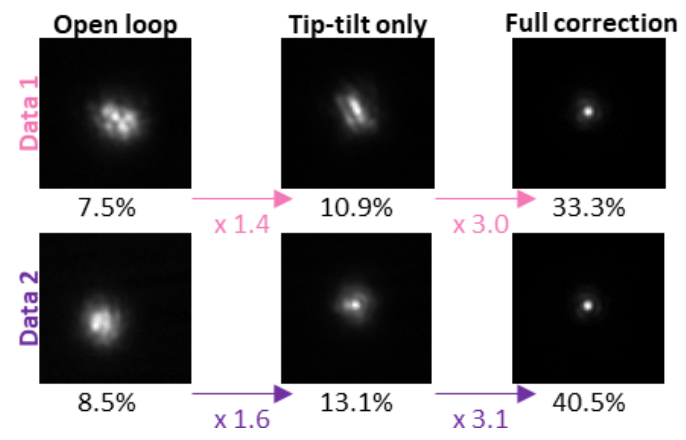

Fig. 6. PSF for no AO correction, tip-tilt correction and full AO correction, for data 1 (up) and data 2 (down). Associated Strehl ratio values and gain between two AO correction configurations are detailed below each PSF image.

\section{B. Single-mode fiber coupling}

As described in part II, the SMF coupling efficiency is computed by normalizing the SMF coupled flux by the flux integrated on the simultaneous FPC images.

The diffraction-limited coupling efficiency estimated, in our case of a $23 \%$ pupil central obscuration, is $74 \%$. In comparison, the maximum value of the mean coupling efficiency $\left\langle\rho_{0}\right\rangle$ measured on an internal source at the telescope focal point was $\left\langle\rho_{0}\right\rangle=67 \%$, which also points out the presence of some NCPAs. Similar to equation (3), the coupling efficiency is described by:

$\rho=\rho_{0} \exp \left(-\sigma_{\text {fitting }}^{2}-\sigma_{\chi}^{2}\right)$

Fig. 7 indicate the expected mean coupling efficiencies for data 1 and data 2 experimental conditions.

On the same figure, the experimental mean coupling efficiencies obtained for data 1 and data 2 for the different AO correction configurations are plotted in crossed dashed lines. Comparing the full AO correction results of the two sets of data, which show similar Strehl ratio and coupling efficiencies close to zero in open loop, the difference of mean coupling efficiency can be attributed to the logamplitude variance term as previously described for the Strehl ratio:

$$
<\rho_{1}>=<\rho_{2}>* \exp \left(-\sigma_{\chi_{1}}^{2}+\sigma_{\chi_{2}}^{2}\right)
$$

where $\left\langle\rho_{i}\right\rangle$ is the mean coupling efficiency of data set $i$. This confirms that the AO loop performance in phase correction is unaffected by the log-amplitude fluctuation in both scintillation conditions. In contrast, the coupling 
efficiency is degraded by the pupil intensity inhomogeneity in the same way the Strehl ratio was shown to be.

Similar to the observations made on the Strehl ratio, the experimental coupling efficiencies with full AO correction are $35 \%$ lower than expected for both data sets. As a first approximation, this is of the same order of the strong NCPAs (especially defocus) described before, and is thus attributed to these NCPAs. Further theoretical estimations of the AO performance and resulting coupling efficiency using our pseudo-analytical modeling tool SAOST [7], [11], [12] will be performed in future work.

The mean coupling efficiency for the medium scintillation data 2 increases from $12.5 \%$ to $29.5 \%$ with the AO correction shifting from tip-tilt only to full correction, i.e. a gain of 2.3. In the case of strong scintillation data 1 , the coupling goes from $6.6 \%$ to $21.8 \%$, i.e. a gain of 3.3 .

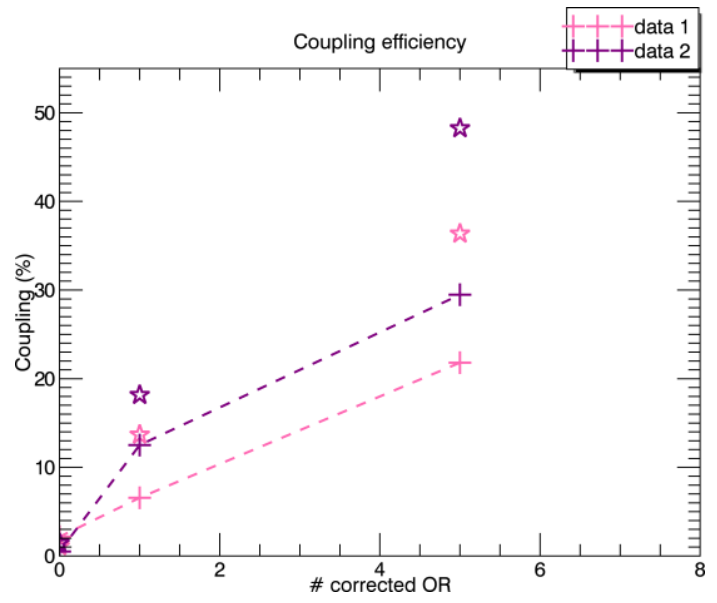

Fig. 7. Mean coupling efficiencies for data 1 and data 2 for different AO corrections (crossed dashed lines) and expected coupling efficiencies (stars).

In Fig. 8, we show the coupling efficiencies temporal variations and histograms of both data sets, acquired with the open loop, tip-tilt only and full AO correction.

To compute the coupling efficiencies time series, the flux integrated on the FPC images acquired at $1 \mathrm{kHz}$ were interpolated to obtain an equivalent FPC flux sampled at $20 \mathrm{kHz}$, which was then then used to normalize the SMF coupled flux (sampled at $20 \mathrm{kHz}$ ). In both cases of scintillation, the coupling efficiency distribution shifts from a decreasing exponential law towards a log-normal one, which is consistent with the theory. The coupling efficiency fluctuation is larger for the strong scintillation data 1 than for data $2\left(\frac{\sigma_{\rho}}{\langle\rho\rangle}=0.39\right.$ and 0.27 , respectively), which is also consistent with the literature. (a) Data 1
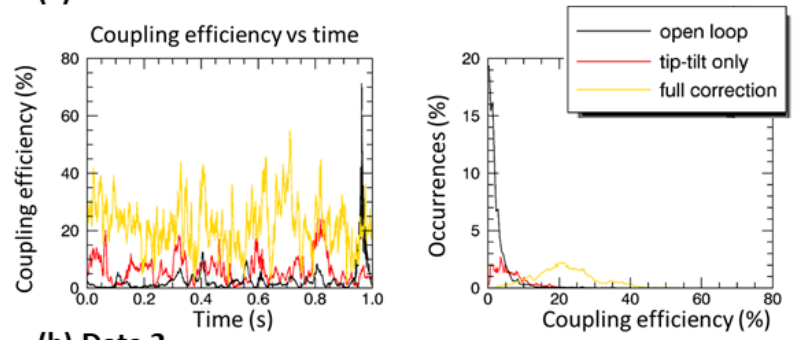

(b) Data 2
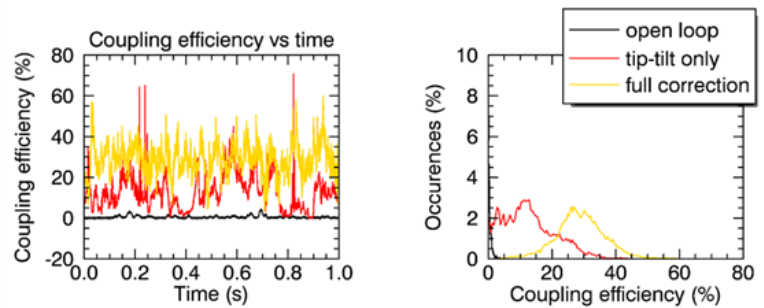

Fig. 8. SMF coupling efficiency and coupling efficiency histogram for (a) data 1 (b) data 2.

\section{CONCLUSION}

In conclusion, we have performed AO-assisted SMF injection of a $1.55 \mu \mathrm{m}$ downlink signal on a $13 \mathrm{~km}$ slant path under various conditions of atmospheric turbulence in the framework of the FEEDELIO experiment. The estimation of the turbulence conditions main parameters (Fried parameter, wind speed, log-amplitude variance) was performed on the WFS data of slopes and intensities. We provided a preliminary analysis of two sets of data both obtained under same $r_{0}$ and wind speed but with different scintillation conditions. As expected for these still reasonable scintillation conditions, the difference in experimental Strehl ratios and coupling efficiencies in full AO correction only lied in the difference in log-amplitude variance. This showed that the phase correction performed by our AO system was not affected by the considered intensity inhomogeneity in the pupil. In contrast, the optical quality (Strehl ratio) and resulting coupling efficiency obtained in full AO correction were still better in the case of weaker scintillation, and were thus degraded by the pupil intensity inhomogeneity. The coupling efficiencies were however $35-40 \%$ lower than expected, which was attributed as a first approximation to NCPAs and especially to a strong defocus aberration. As compared to tip-tilt only, the mean coupling efficiency was multiplied by $>2$ with full AO correction in both cases of scintillation strengths. The strong scintillation impact on the coupling efficiency was mainly: to reduce the gain brought by a full AO correction compared to a tip-tilt only, and to widen the coupling efficiency distribution probability (i.e. to strengthen the fluctuations amplitude). These results are consistent with the analysis performed on the uplink pre-compensated fiber coupling presented in [13]. This work will be complemented by analytical modeling of SMF coupling in the encountered turbulence conditions, and by further processing of the large variety of data acquired during this project. 


\section{ACKNOWLEDGMENT}

Thanks are due to G. Artaud, Zoran Zodnik and Christoph Voland for fruitful discussions and technical support. This work was supported by the ESA contract $\mathrm{N}^{\circ} 4000120300 / 17 / \mathrm{NL} / \mathrm{PS}$ and by the CNES R\&T DOCIL. The authors also thank the technical staff of the Instituto Astrofísica de Canarias, Teide's National Park and Teide's cable car station for their essential technical support.

\section{REFERENCES}

[1] R. K. Tyson, J. S. Tharp, et D. E. Canning, « Measurement of the bit-error rate of an adaptive optics, free-space laser communications system, part 2: multichannel configuration, aberration characterization, and closed-loop results », Optical Engineering, vol. 44, no 9, p. 096003 , sept. 2005.

[2] T. Berkefeld, D. Soltau, R. Czichy, E. Fischer, B. Wandernoth, et Z. Sodnik, "Adaptive optics for satellite-to-ground laser communication at the $1 \mathrm{~m}$ Telescope of the ESA Optical Ground Station, Tenerife, Spain », Proc. SPIE, vol. 77364, p. 77364C, juill. 2010.

[3] K. E. Wilson et L. C. J. Roberts, «Recent Developments in Adaptive Optics for the LCRD Optical Ground Station at Table Mountain », Proc. ICSOS, p. 5, 2014

[4] M. W. Wright, J. F. Morris, J. M. Kovalik, K. S. Andrews, M. J. Abrahamson, et A. Biswas, « Adaptive optics correction into single mode fiber for a low Earth orbiting space to ground optical communication link using the OPALS downlink », Optics Express, vol. 23, $\mathrm{n}^{\mathrm{o}} 26$, p. 33705 , déc. 2015.

[5] C. Petit et al., «Investigation on adaptive optics performance from propagation channel characterization with the small optical transponder », Optical Engineering, vol. 55, $\mathrm{n}^{\mathrm{o}}$ 11, p. 111611, sept. 2016.

[6] A. M. Bonnefois et al., "Adaptive optics precompensation for GEO feeder links: the FEEDELIO experiment ", in International Conference on Space Optics - ICSO 2018, 2019, vol. 11180, p. 111802C.

[7] N. Védrenne, J.-M. Conan, C. Petit, et V. Michau, « Adaptive optics for high data rate satellite to ground laser link », Proc. SPIE, p. 97390E, 2016.

[8] J.-M. Conan, G. Rousset, et P.-Y. Madec, « Wave-front temporal spectra in high-resolution imaging through turbulence ", J. Opt. Soc. Am. A, vol. 12, n 7 , p. 1559, juill. 1995.

[9] N. Vedrenne, J.-M. Conan, A. Bonnefois, C. Petit, M.T. Velluet, et V. Michau, "Adaptive optics precompensation for GEO feeder links: Towards an experimental demonstration ", in 2017 IEEE International Conference on Space Optical Systems and Applications (ICSOS), Naha, 2017, p. 77-81.

[10] R. J. Sasiela, «Basic Equations for Wave Propagation in Turbulence ", in Electromagnetic Wave Propagation in Turbulence, vol. 18, Berlin, Heidelberg: Springer Berlin Heidelberg, 1994, p. 19-46.

[11] L. Canuet et al., « Statistical properties of single-mode fiber coupling of satellite-to-ground laser links partially corrected by adaptive optics ", Journal of the Optical Society of America A, vol. 35, n 1, p. 148, janv. 2018.

[12] J.-M. Conan et al., "Adaptive optics for ground GEO-satellites optical links: from system design to experimental demonstration », Proc. of AOIM XII Conference, in press, 2019.

[13] N. Vedrenne et al., « First experimental demonstration of adaptive optics pre-compensation for GEO feeder links in a relevant environment », Proc. ICSOS 2019 (submitted), 2019. 